\title{
Recurrent genital candidosis in women and the effect of intermittent prophylactic treatment
}

\author{
FIONA DAVIDSON AND R. F. MOULD \\ From the Department of Sexually Transmitted Diseases and the Department of Medical Physics, Westminster \\ Hospital
}

SUMMARY A study of clotrimazole for the treatment of recurrent genital candidosis unexpectedly showed that symptoms and infection can be dissociated. The aim of the study was to see if intermittent antifungal treatment would reduce symptoms in women constantly distressed by recurrent genital candidosis. Forty women seriously affected by the condition were initially given oral and local antifungal treatment. When the patients were symptom-free and the vagina was free of yeasts, they were entered into a double-blind clinical trial and were treated prophylactically for four months with either intermittent clotrimazole pessaries and cream or a placebo. The prophylactic treatment kept symptoms below a critical level but did not affect the return of the yeasts to the vagina. This dissociation between symptoms and vaginal yeasts was unexpected. Rectal yeast carriage was unaffected by prophylactic vaginal treatment. Male contacts and patients both showed a high incidence of non-specific genital infection. This association has seldom been reported. A few patients cultured yeasts from their homes but this environment was not considered a major source of reinfection. The vaginal $\mathrm{pH}$ did not appear to be altered by the presence of yeasts. The results of the study suggested that symptoms in women with recurrent genital candidosis were not caused by yeasts alone, and possibly the reason for recurrences might lie not in constant reinfection by yeasts, but in failure to recognise and remove a primary underlying factor, perhaps infection with other sexually transmitted agents. The question of a synergistic action between yeasts and other organisms is discussed.

\section{Introduction}

Yeast infection of the genital and perianal area in women is common (Hurley et al., 1973; Hilton and Warnock, 1975; Willmott, 1975) and according to the report of the Chief Medical Officer for 1975 (Department of Health and Social Security, 1976) the incidence is increasing. This is said to be enhanced by such factors as antibiotics, immunosuppressive drugs, pregnancy, and diabetes (Gardner and Kaufman, 1969), while even nylon tights have been implicated (Bull, 1969).

There is however a group of women prone to recurrent attacks of genital and perianal candidosis in whom no obvious provoking factor can be found. These patients have recurring attacks at varying intervals, often for many years causing much distress

Presented in part as a Free Communication at the Eighth World Congress of Gynaecology and Obstetrics in Mexico, October 1976

Address for reprints: Dr F. Davidson, Westminster Hospital, Department of Sexually Transmitted Diseases, Dean Ryle Street, London SW1P 2AP

Received for publication 11 July 1977 to themselves and their sexual partners. This study was therefore undertaken to find the effect of intermittent prophylactic treatment in this group of women.

\section{Patients and methods}

Forty women attending the Westminster Hospital Department of Sexually Transmitted Diseases between 1974 and 1976 were selected for the trial. The criterion for their inclusion was that they had either a history of recurrent 'thrush' for at least one year treated elsewhere each time with antifungal therapy, and confirmed where possible, or had had at least three proved treated clinical attacks of candidosis in the previous six months in the clinic.

In all cases the patients had distressing symptoms of itching or discharge, signs of vulvitis or vaginitis (Carroll et al., 1973), and yeasts were grown on culture. At each visit oral, vaginal, and rectal swabs were taken with polyester sponge swabs (Oates et al., 1971). The oral swabs were taken from both sides of the buccal mucosa opposite the first molar tooth. 
The vaginal swab was taken from the secretions in the posterior fornix and midvaginal epithelium, and a swab was taken from the rectal mucosa through a proctoscope. Smears were stained by Gram's method and examined microscopically for yeasts, and the swabs were cultured in Feinberg-Whittington medium at $37^{\circ} \mathrm{C}$ for 48 hours. The yeasts cultured were further identified by the germ tube method and sugar fermentation. The $\mathrm{pH}$ of the vaginal discharge was measured at each visit with simple narrow range litmus paper* accurate to $\mathbf{0 . 5}$.

As the patients selected had to attend regularly for a year, only those who were seriously distressed by the problem, who appeared reliable, were cooperative, and lived reasonably near were included in the trial. It was hoped these precautions would reduce defaulting to the minimum. A schematic diagram of the trial is shown in Fig. 1.

All the patients were treated initially with a standard regimen, consisting of one oral nystatin tablet three times a day for 14 days, and one vaginal clotrimazole pessary each night, with clotrimazole cream to the genital and perianal area for 12 consecutive nights. The patients were instructed exactly how to use the pessaries and cream; to use them during menstruation if it occurred and without internal tampons. They were also told not to douche or wash out the vagina, use any additives to their baths, nor use any vulvovaginal deodorants.

One week after finishing this treatment the patients were re-examined. This was the assessment point for entering the trial. If symptoms had subsided and the
Gram-stained smear was negative for yeasts, while waiting for the culture result, the patient was given a code number which placed her randomly in one of the two groups. The code was held by the pharmaceutical company ${ }^{\prime}$ which provided the drugs, and was not broken until the end of the trial.

The first group was given active clotrimazole pessaries and cream for prophylactic use. They had four special packs each containing six pessaries and one tube of cream. The second group was given similar packs of placebo pessaries and cream. The active treatment was identical in appearance with the placebo treatment, which consisted of the inactive base. None of the patients knew that there was placebo treatment. Ethical approval was given for this study by the appropriate committee.

Each patient was given an emergency pack containing six active clotrimazole pessaries and a tube of clotrimazole cream for use only if severe symptoms developed between follow-up visits and she could not attend the clinic.

The patients used the follow-up treatment of one pessary and cream each night from the fifth to eleventh day inclusive of the next four menstrual periods. They returned for examination two weeks after the end of each treatment, just before the next period was due. It was at this time that relapse was considered most likely to occur.

At each visit they were asked whether they had used the treatment or had needed the emergency

*Whatman Biochemicals

† Bayer UK Ltd

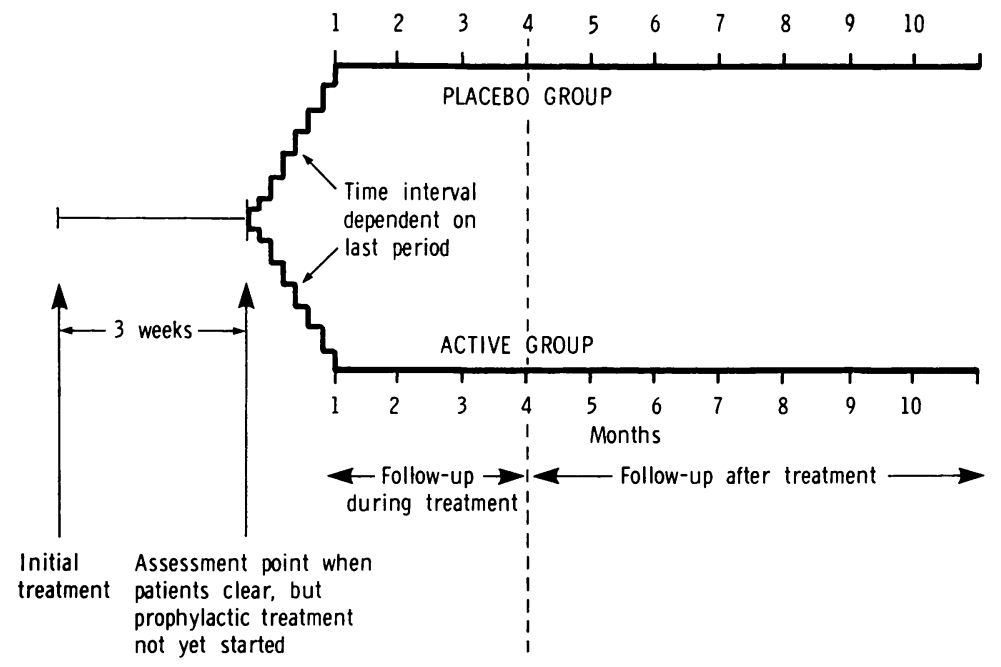

Fig. 1 Schematic diagram of the trial. 
pack. At the end of the four months' treatment they continued to return monthly in the same way for a further six months.

Each patient was also given two Sabouraudchloromycetin culture plates to expose at home, one in the bathroom and one in the bedroom, to see if Candida species would grow from the atmosphere.

\section{Results}

Forty women entered the trial with 20 allocated to the active treatment and 20 to the placebo treatment. Table 1a and $\mathrm{b}$ shows that both groups were similar in terms of age, duration of recurrent attacks of 'thrush', and duration of the presenting attack. One patient in the placebo group was diabetic.

As some of the patients had attended other clinics for previously proved attacks of 'thrush' the range of number of proved attacks treated at the Westminster Hospital clinic varied in both groups from two to 13 .

\section{SYMPTOMS}

As the trial set out to evaluate the effect of a prophylactic treatment for four months, all the patients were free from severe symptoms before beginning the treatment under study. Figure 2 shows the effect of this treatment on severe symptoms for the two groups. It illustrates when each patient either

Table 1a Data for the two groups of patients in the trial

\begin{tabular}{|c|c|c|c|c|c|c|c|}
\hline \multirow[t]{2}{*}{ Group } & \multirow[t]{2}{*}{$\begin{array}{l}\text { No. of patients } \\
\text { in group }\end{array}$} & \multicolumn{2}{|c|}{ Ages of patients (years) } & \multicolumn{2}{|c|}{$\begin{array}{l}\text { Duration of recurrent } \\
\text { 'thrush' (years) }\end{array}$} & \multicolumn{2}{|c|}{ Duration of presenting attack (days) } \\
\hline & & Mean & Range & Mean & Range & Mean & Range \\
\hline $\begin{array}{l}\text { Active } \\
\text { Placebo }\end{array}$ & $\begin{array}{l}20 \\
20\end{array}$ & $\begin{array}{l}25 \\
26\end{array}$ & $\begin{array}{l}(20-30) \\
(19-43)\end{array}$ & $\begin{array}{l}3 \\
3\end{array}$ & $\begin{array}{l}(0.5-9) \\
(0.5-8)\end{array}$ & $\begin{array}{l}27 \\
29\end{array}$ & $\begin{array}{l}(3-140) \\
(2-168)\end{array}$ \\
\hline
\end{tabular}

Table 1b Data for the two groups of patients in the trial

\begin{tabular}{llll}
\hline Group & $\begin{array}{l}\text { No. of proved attacks of yeast infection } \\
\text { up to time of trial in clinic }\end{array}$ & $\begin{array}{l}\text { No. of patients treated } \\
\text { previously with clotrimazole }\end{array}$ \\
\cline { 2 - 4 } & Mean & Range & $\begin{array}{l}\text { No. of patients with known } \\
\text { non-specific infection during trial }\end{array}$ \\
\hline Active & $4 \cdot 2$ & $(2-13)$ & 17 \\
Placebo & $4 \cdot 3$ & $(2-13)$ & 17 \\
\hline
\end{tabular}

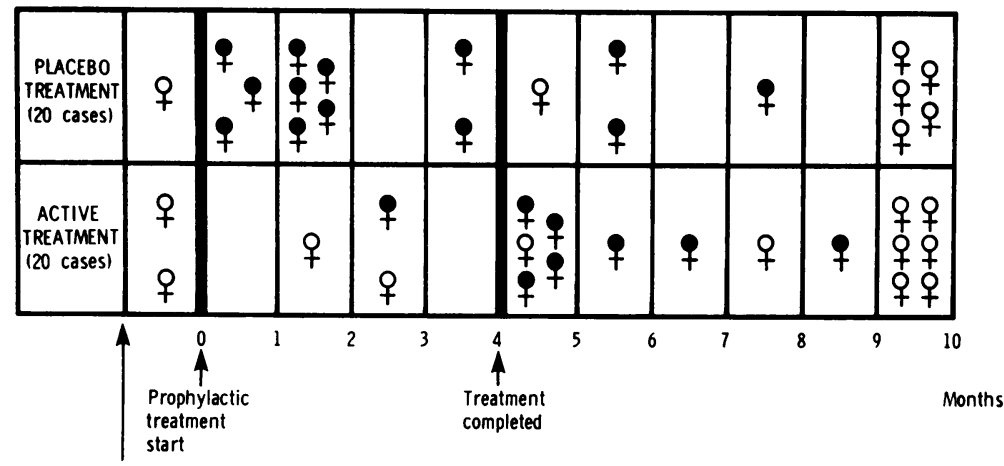

Assessment point when

patients clear, but

prophylactic treatment

not yet started

$$
\text { Notation: } \begin{aligned}
q & =\text { Symptoms so severe that treatment required } \\
q & =\text { No symptoms, but patient lost to follow-up }
\end{aligned}
$$

Fig. 2 Follow-up diagram of all patients in the trial, indicating when symptoms became so severe that treatment was required. 
developed symptoms so severe that retreatment was required or, if she did not complete the course, when she was last seen free from symptoms. For example, in the placebo group one patient free from symptoms was 'lost' before the prophylactic treatment was started and another was last seen free from symptoms after four months. In the active group, two patients were 'lost' before treatment began and one each was last seen free from symptoms after one, two, four, and seven months. This was in spite of every possible effort made to get the patients to attend.

Symptoms have been considered only when severe enough for retreatment as terms such as itching and discharge were thought to be too subjective. Any patient who had used her emergency pack before her visit was considered to have had symptoms severe enough for retreatment even if at the next visit she was then asymptomatic, or the vagina showed no yeasts.

Because of the loss of patients before treatment, the numbers available for statistical analysis were reduced to 19 in the placebo group and 18 in the active group. The most notable feature of Fig. 2 is the pattern during the first four months. During this period 10 of the 19 patients in the placebo group demonstrated symptoms so severe that they required retreatment during this first four months but only one of the 18 patients in the active group required retreatment during this period. This is statistically highly significant, $\mathbf{P}<\mathbf{0 . 0 1}$. However, the active group contained two patients lost to follow-up during this time but even if it is assumed that one of these exhibited severe symptoms before the four months elapsed, the difference between the two groups is still statistically significant (Table 2 ).

After prophylactic treatment stopped, the patients in the active group began to relapse and required retreatment. At the last follow-up at 10 months, five of the 19 patients in the placebo group and six of the 18 patients in the active group were still symptom-free. However, they are not directly comparable because of the different pattern of follow-up

Table 2 Contingency table relating symptoms after treatment in the placebo and active treatment groups

\begin{tabular}{|c|c|c|}
\hline \multirow{2}{*}{ Group } & \multicolumn{2}{|c|}{ Symptoms during 4 months' treatment } \\
\hline & Severe symptoms & No severe symptoms \\
\hline $\begin{array}{l}\text { Placebo } \\
\text { Active }\end{array}$ & $\begin{array}{r}10 \\
2\end{array}$ & $\begin{array}{r}9 \\
16\end{array}$ \\
\hline
\end{tabular}

With Yates's correction $\chi_{1}^{2}=5.51 \mathrm{P}=0.019$.

The data for this table were obtained from Fig. 2. In the active group, 2 patients were lost to follow-up during the four months of treatment while asymptomatic. An assumption is made that on average half those lost to follow-up would have experienced severe symptoms. Thus, in the contingency table the numbers 2 and 16 refer to the active group. losses in the two groups. Seven patients in the placebo group and one patient in the active group had used their emergency packs by the end of the first four months. Most of the emergency packs were used in the first two months of the trial, and most of the failures in the active group were in the first two months after all treatment had ceased, indicating that symptoms were kept under control by the clotrimazole.

\section{CULTURES}

Figure 3 shows the return of the yeasts to the vagina, regardless of symptoms, for the two groups of patients. There is no significant difference between the results of the two groups, $\mathrm{P}>0.5$, Table 3 .

The presence of symptoms, and positive yeast culture results at each patient-visit were recorded (Table 4). In both groups severe symptoms occurred with negative culture results and positive results occurred without symptoms. There was no definite relationship between time to reappearance of yeasts and time to symptoms.

\section{RECTAL YEASTS}

Fewer than half of the patients with recurrent genital candidosis carried rectal yeasts initially, six out of 19 and eight out of 19 in the two groups. After oral nystatin only one of the six in the first group and two of the eight in the second group still grew yeasts. However, during the four months after oral nystatin the yeasts returned and at the end of this time the number with rectal yeasts was slightly higher than initially, eight out of 19 and 10 out of 19 in the two groups. There was no difference in the rectal yeast carriage rate during the prophylactic treatment between the active and placebo groups.

\section{ORAL YEASTS}

Thirty-two of the original 40 patients had oral yeasts before treatment and 26 of these had oral yeasts after two weeks' treatment with oral nystatin. There

Table 3 Contingency table relating regrowth of yeasts in the vagina after treatment in the placebo and active treatment groups

\begin{tabular}{lll}
\hline \multirow{2}{*}{ Group } & \multicolumn{1}{c}{ Vaginal yeasts during } & 4 month' treatment \\
\cline { 2 - 3 } & Yeasts regrew & No yeasts grown \\
\hline Placebo & 10 & 9 \\
Active & 9.5 & $7 \cdot 5$ \\
\hline
\end{tabular}

With Yates's correction $\chi_{1}{ }^{2}=0.10 \mathrm{P}>0.5$.

The data for this table were obtained from Fig. 3. In the active group, three patients were lost to follow-up during the four months of treatment while asymptomatic. An assumption is made that on average half those lost to follow-up would have regrown yeasts and half would not. Thus, in the contingency table the numbers 9.5 and 7.5 refer to the active group. 
were insufficient patients either without oral yeasts or without orogenital contact to make any comparisons in this study.

\section{CONTACTS}

Information was available concerning 36 male contacts of 32 patients. Thirty-two contacts were seen in the Westminster Clinic and four contacts were seen in other clinics. Four patients had two contacts, two patients had no contacts, and six patients had contacts who refused to attend. Twentyfour contacts had coronal and meatal swabs taken of which $10(42 \%)$ grew yeasts, and five contacts had symptoms of balanitis but were not seen at the clinic. Thirty-three of the total 42 contacts used clotrimazole cream to reduce the possibility of

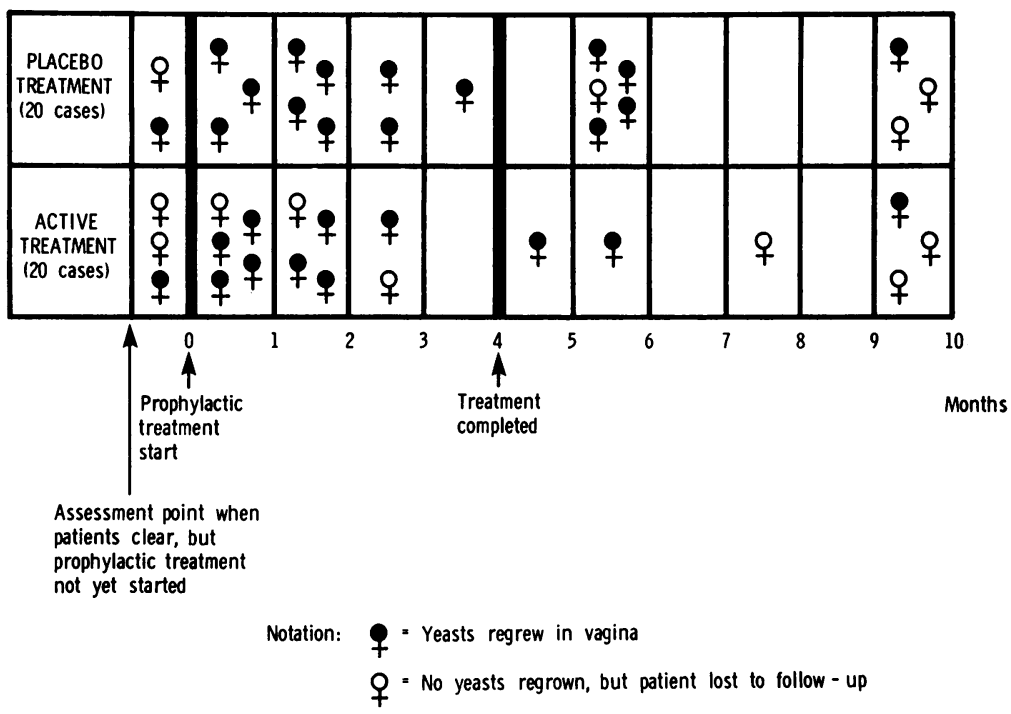

Fig. 3 Follow-up diagram of all patients in the trial, indicating when yeasts grew in the vagina.

Table 4 Results for symptoms and reappearance of yeasts

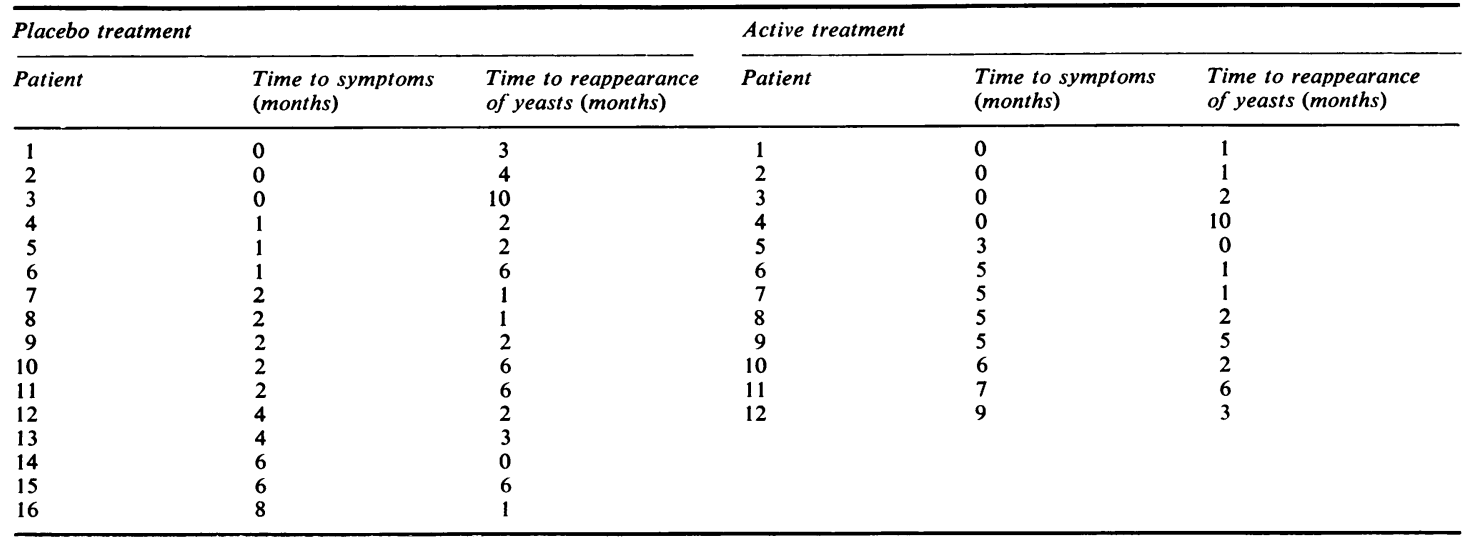

Placebo group

One patient was lost to follow-up

Two patients had no symptoms and also yeasts did not reappear

One patient had no symptoms, but yeasts reappeared two weeks before start of placebo treatment Active treatment group

Two patients were lost to follow-up

Five patients had no symptoms and also yeasts did not reappear

One patient had no symptoms, but yeasts reappeared two weeks before start of placebo treatment 
reinfection. The other nine refused treatment. Twenty male contacts had non-specific urethritis: several were asymptomatic and in three this was associated with genital warts. A further five contacts had a history of non-specific urethritis while associating with the patients. Thus $25(70 \%)$ of the 36 male contacts seen had non-specific genital infection during their association with the patients with recurrent genital candidosis.

Twelve $(63 \%)$ patients in the active group and 14 $(74 \%)$ patients in the placebo group had had contact with a patient with non-specific urethritis or were considered to have non-specific infection diagnosed from signs of cervicitis, urethritis, or salpingitis in the absence of known pathogens. Some of the male contacts of these patients however were not seen.

Thus, contacts and patients had a high incidence of non-specific genital infection.

\section{CONTRACEPTION}

Seventeen of 20 in the active group and 10 of 20 in the placebo group were taking the contraceptive pill.

\section{VAGINAL pH}

The vaginal $\mathrm{pH}$ as measured by the narrow range $\mathrm{pH}$ paper, before and after treatment in the patients with recurrent candidosis, was compared with a control group with no evidence of yeast infection. There was no difference between the groups. The vaginal $\mathrm{pH}$ was remarkably constant for individual patients. Variations occurred with the presence of blood or after coitus.

\section{ENVIRONMENT}

Twenty-eight of the 40 patients returned their bathroom and bedroom culture plates. Eleven of the 56 plates grew Candida species (five Candida albicans and six Candida guillermondi). These organisms were isolated from the corresponding patients. No $C$. albicans was grown from a group of 21 normal controls,* only two of 42 plates grew Candida species, one grew Candida parapsilosis and the other C. guillermondi.

\section{YEAST IDENTIFICATION}

Thirty-two of the $\mathbf{4 0}$ patients grew $C$. albicans from the vagina, five of these with $C$. guillermondi or Torulopsis glabrata. When yeasts were isolated from mouth, vagina, and rectum, $C$. albicans was usually grown, although occasionally the cultures from these sites differed from each other. The yeasts in the remaining eight patients were not identified.

*Normal controls were culture plates taken home by staff from the clinic. These people had no known complaints but were not examined

\section{Discussion}

Odds (1977) remarked that an antifungal preparation should be expected to eliminate only the yeasts from the female genital tract. Its success in relieving symptoms depends on those symptoms being caused exclusively by the yeasts. In the trial of antifungal treatment it was expected that the reduction in symptoms would be associated with the reduction in yeasts cultured from the vagina. Therefore, the observed dissociation between symptoms and positive yeast cultures was a surprise. The intermittent clotrimazole treatment undoubtedly reduced symptoms in the patients with recurrent genital candidosis, which was the original aim, but therapy did not affect regrowth of yeasts in the vagina. It could be argued that symptoms were associated with the number of yeast colonies present and that intermittent treatment simply reduced the total colony count below a critical level. However, Mursic (1975) found that patients with or without symptoms could have the same yeast count.

One possibility is that clotrimazole was acting locally on some factor other than the fungus alone. When the treatment stopped, the symptoms returned in many patients but were not always accompanied by the fungus. Mendel and Haberman (1965) commented on this aspect. They studied patients with cultural and clinical cures who returned later with recurrent symptoms, although the cultures were often still negative. They noticed that clinical cures and alleviation of symptoms occurred during therapy when the organisms were still present, and that in some cases the same fungal treatment could relieve those with symptoms in whom no Candida was ever isolated.

These observations suggest Candida is always a secondary invader in the vagina, either combining with an underlying factor to produce symptoms or enhancing the symptoms this factor has already produced. Gardner and Kaufman (1969) referred to this underlying factor as Factor $X$.

C. albicans as a primary infecting organism does not really fulfil Koch's postulates (Koch, 1890). It can occur in the vagina without signs or symptoms of disease and the classical signs and symptoms of candidal vaginitis may occur without the isolation of the yeasts (Carroll et al., 1973). Maibach and Kligman (1962) found it impossible to infect a healthy subject with Candida. Damage was always needed first.

However, one of the most striking features among the patients in the trial and their contacts was the high incidence of non-specific infection. Seventy per cent of the male contacts had non-specific infection during their association with the patients 
in the trial. In several cases the male contacts of the trial patients were asymptomatic and the urethritis would have been missed if not looked for carefully. A high proportion of the men carried yeasts on the penis confirming earlier results (Davidson, 1977). Rohatiner (1966) found a similar association with non-specific urethritis and he commented on the possibility of a relationship between Candida and other organisms found in the male or female genital tract. Loh and Baker (1955) while investigating the effect of tetracycline on yeasts in the gut noticed there was a longer and more persistent overgrowth with Proteus species. Then Yamabayashi (1958) noticed that $C$. albicans enhanced the virulence of Proteus vulgaris, Pseudomonas aeruginosa, and to a lesser extent Escherichia coli in experimental mice but it did not enhance the growth of these organisms in vitro, nor did it affect the virulence or growth of Staphlococcus aureus, Bacillus subtilis, or Streptococcus haemolyticus. Mendel and Haberman (1965) noticed a strong correlation, in their patients, between positive Candida cultures and the presence of $E$. coli and A. aerogenes in the vagina. Söltz-Szöts and Thurner (1969) said pure cultures of Candida were almost never found in the male urethra. They considered that Candida could only multiply in the urethra if, together with the Candida, there was irritation of the mucosa by trauma, bacteria, viruses, or Trichmomonas vaginalis. They also noted candidal balanitis was often associated with massive bacterial colonisation. Willmott (1975) noticed $34 \%$ of his patients with yeasts had other infections.

The possible association of yeasts with nonspecific genital infection produces many problems. The causes of non-specific infection are as difficult to define as the causes of candidal vaginitis. The most persistently implicated aetiological agents in non-specific infection are Chlamydia trachomatis and the mycoplasmas but no pathognomic signs or symptoms need occur in either sex, and they may be asymptomatic.

The possibility of reinfection of the vagina with yeasts from the rectum raises another unanswered question. There is a known association between Candida of the rectum and Candida of the vagina (Anyon et al., 1971), but this does not necessarily infer a causal relationship between them. Not all the patients in the trial had yeasts in the rectum and certainly the results showed no evidence that the vagina was infected from the rectum or vice versa. Candida was found to occur in the homes of a few of the patients but the environment was not considered a major contributory factor in these recurrences. The acidity of the vagina in those patients with recurrent genital candidosis was remarkably constant and did not differ from those with no yeast problem. Similar results were found by Cohen (1969).

More patients in the active treatment group than the placebo group used oral contraceptives, but as yeast regrowth was the same in both groups and the symptoms were less in the active group, there is no evidence that oral contraceptives had any marked influence on these patients.

It has long been stated that Candida is a disease of the diseased. Wilson (1962) believed that it could even indicate underlying disease before this was clinically detectable. Kirkpatrick et al. (1971) considered that treatment directed only to the organism could be predictably inadequate since the 'fertile soil' for Candida persisted in deficient hosts even after elimination of the organism. As a consequence, although the remission with chemotherapy might be complete, it was almost always temporary. The rational approach should be towards the host and not the organism.

It may be that the common factor necessary is some disorganisation of skin or mucous membrane at a cellular level which allows the fungus to become pathogenic. Simple remedies such as preventing self damage might be sufficient-for example, no douching, no vaginal deodorants, no bubble baths. Perhaps the use of simple lubricants such as KY Jelly* in sexual intercourse for those who do not lubricate well, might prevent mechanical damage. However it is most important to consider always the possibility of underlying infections in women with recurrent genital candidosis and in their contacts.

We are very grateful to Dr J. K. Oates for permission to investigate his patients, and to Mr N. Richardson and $\mathrm{Mr}$ M. Y. Imambaccus for their expert technical assistance. We would like to thank Bayer Pharmaceuticals (UK) Ltd for their assistance and for supplying clotrimazole. We are also grateful to Mrs V. S. Chapman for secretarial help.

*Johnson and Johnson Ltd

\section{References}

Anyon, C. P., Desmond, F. B., and Eastcott, D. F. (1971). A study of Candida in one thousand and seven women. New Zealand Medical Journal, 73, 9-13.

Bull, M. J. V. (1969). Letter: Wearing tights. British Medical Journal, $1,120$.

Carroll, C. J., Hurley, R., and Stanley, V. C. (1973). Criteria for diagnosis of Candida vulvovaginitis in pregnant women. Journal of Obstetrics and Gynaecology of the British Commonwealth, 80, 258-263.

Cohen, L. (1969). Influence of pH on vaginal discharges. British Journal of Venereal Diseases, 45, 241-246.

Davidson, F. (1977). Yeasts and circumcision in the male. British Journal of Venereal Diseases, 53, 121-122.

Department of Health and Social Security (1976). Report of the Chief Medical Officer on the State of the Public Health for the Year 1975, pp. 47-51. HMSO: London. 
Gardner, H. L., and Kaufman, R. H. (1969). Candidiasis (moniliasis). In Benign Diseases of the Vulva and Vagina, first edition, pp. 149-167. Mosby: St Louis.

Hilton, A. L., and Warnock, D. W. (1975). Vaginal candidiasis and the rôle of the digestive tract as a source of infection. British Journal of Obstetrics and Gynaecology, 82, 922-926.

Hurley, R., Leask, B. G. S., Faktor, J. A., and de Fonseka, C. I. (1973). Incidence and distribution of yeast species and Trichomonas vaginalis in the vagina of pregnant women. Journal of Obstetrics and Gynaecology of the British Commonwealth, 80, 252-257.

Kirkpatrick, C. H., Rich, R. R., and Bennett, J. E. (1971). Chronic muco-cutaneous candidiasis: Model-building in cellular immunity. Annals of Internal Medicine, 74, 955-978.

Koch, R. (1890). An address on bacteriological research. British Medical Journal, 2, 380-383.

Loh, W. P., and Baker, E. E. (1955). Fecal flora of man after oral administration of chlortetracycline or oxytetracycline. Archives of Internal Medicine, 95, 74-82.

Maibach, H. I., and Kligman, A. M. (1962). The biology of experimental human cutaneous moniliasis (Candida albicans). Archives of Dermatology, 85, 233-257.

Mendel, E. B., and Haberman, S. (1965). The vaginal ecology and its relationship to symptoms in vaginitis. Southern Medical Journal, 58, 374-378.
Mursic, P. V. (1975). A diagnosis, pathogenicity and therapy of candidiasis. Münchener medizinische Wochenschrift, 117, 893-896.

Oates, J. K., Selwyn, S., and Breach, M. R. (1971). Polyester sponge swabs to facilitate examination for genital infection in women. British Journal of Venereal Diseases, 47, 289-292.

Odds, F. C. (1977). Trends in candidal vaginitis. Proceedings of the Royal Society of Medicine, 70, Supplement 4, 24-28.

Rohatiner, J. J. (1966). Relationship of Candida albicans in the genital and anorectal tracts. British Journal of Venereal Diseases, 42, 197-200.

Söltz-Szöts, J., and Thurner, J. (1969). Budding fungi infection of the male and female sexual organs. Zeitschrift für Haut-und Geschlechtskrankheiten, 44, 545-548.

Willmott, F. E. (1975). Genital yeasts in female patients attending a VD clinic. British Journal of Venereal Diseases, 51, 119-122.

Wilson, J. W. (1962). The biology of experimental human cutaneous moniliasis (Candida albicans). Archives of Dermatology, 85, 233-257.

Yamabayashi, H. (1958). A zymosanlike substance extracted from Candida albicans. Medical Journal of Osaka U'niversity, 9, 11-21. 